\title{
Desigualdad y Políticas Sociales Erróneas Producen Inequidad en México
}

Inequality and erroneous social policy produce inequity in México

\author{
Catalina Eibenschutz ${ }^{1}$, Silvia Támez ${ }^{1}$ e Iliana Camacho²
}

\begin{abstract}
1 Departamento de Atención a la Salud. Área de investigación Estado y servicios de salud. Universidad Autónoma Metropolitana. México, D.F. ceibenschutz@yahoo.com.mx, stamez@correo.xoc.uam.mx

2 Universidad Autónoma Metropolitana, México, D.F. E-mail: ilia_cuapio@yahoo.com
\end{abstract}

Recibido 8 Enero 2007/Enviado para Modificación 2 Mayo 2008/Aceptado 26 Agosto 2008

\section{RESUMEN}

Se discuten diferentes conceptualizaciones de equidad y desigualdad en salud, concluyendo que inequidad y desigualdad no son sinónimos, y se sugiere la utilización de desigualdad para describir perfiles epidemiológicos y equidad en relación con la distribución y el acceso de los servicios de atención a la salud. Se revisa en más detalle la situación de México, donde existe gran polarización socioeconómica, una reforma sanitaria privatizadora que ha aumentado la inequidad y una ciudadanía incompleta, como obstáculos principales para conseguir una disminución de la inequidad en salud.

Palabras Clave: Inequidad en salud, inequidad social, política de salud, sistemas de salud, participación ciudadana, derecho a la salud, desigualdades en la salud (fuente: DeCS, BIREME).

\section{ABSTRACT}

The different conceptualisations regarding equity and inequality in health are discussed, concluding that inequity and inequality are not synonyms. It is suggested that inequality should be used for describing epidemiological profiles and equity in relationship to the distribution of and gaining access to health attention services. The situation in México is reviewed in greater detail, as there is great socioeconomic polarisation in this country, a privatising sanitary reform which has increased inequity and incomplete citizenry as the main obstacles for gaining a reduction in inequity in health.

Key Words: Inequity in health, social inequity, health policy, health system, citizen participation, the right to health, inequalities in health (source: MeSH, NLM). 
$\mathrm{E}$ l objetivo de este trabajo es discutir los conceptos de equidad en salud y plantear la diferencia que, en nuestra opinión, existe entre los conceptos de inequidad y desigualdad. Además, queremos demostrar la hipótesis de que en países como México, con una polarización social extrema y un Estado brutalmente “delgado”, el discurso “moderno" de la equidad en salud no pasa de ser un discurso de carácter retórico.

Respecto al concepto equidad, existe una gran variedad de definiciones, pues es motivo de estudio de distintas disciplinas. Esta situación muestra la complejidad del tema y la dificultad para delimitarlo. Sin embargo, nuestra finalidad no es profundizar en una serie de definiciones de equidad, sino señalar qué tan útil es seguir hablando de equidad en las sociedades capitalistas periféricas, si bajo el modelo neoliberal y las políticas de ajuste han aumentado la polarización socioeconómica y política dentro de sus respectivos países.

Cualquier idea bajo la que se conciba la equidad en salud refleja una responsabilidad ético-política frente a la desigualdad en salud; significa también una idea precisa sobre la justicia social que podríamos definir, en términos de equidad en salud, como la necesidad de ofrecer y garantizar más servicios a los que peor salud tienen. Esta simple idea de justicia social suena imposible de ser cumplida bajo una estructura capitalista neoliberal de cualquier sociedad periférica como las latinoamericanas.

La perspectiva de los organismos intergubernamentales

En los últimos 20 años, la inequidad y la desigualdad han sido motivo de una gran preocupación en las instituciones multilaterales como el Banco Mundial (BM) y la Organización Mundial de la Salud (OMS), justamente después de la adopción de las políticas de corte neo-liberal en todo el mundo subdesarrollado.

Desde la perspectiva de la OMS, equidad es "el logro por parte de todas las personas del bienestar más alto alcanzable en contextos específicos. Consecuentemente, la equidad en la atención de salud significaría que los recursos para la salud sean asignados de acuerdo con la necesidad, que los servicios sean recibidos de acuerdo con las necesidades y que la contribución al financiamiento de los mismos sea hecho de acuerdo con la capacidad económica” (1).

Como todas las definiciones de OMS, hace referencia a contextos específicos, lo cual significa que hay países que se pueden permitir "máximos de bienestar" y consecuentemente los países pobres buscan "mínimos de bienestar”. 
Revisamos la perspectiva del Banco Mundial desde el Informe sobre el Desarrollo Mundial 2006 (2), cuyo tema central fue precisamente Equidad y Desarrollo; en este documento argumentan: «cuando hablamos de equidad nos referimos a que todas las personas deberían tener las mismas oportunidades para orientar su vida en la forma que ellas mismas decidan y estar a salvo de privaciones graves en cuanto a las consecuencias». Bajo esta lógica, la relación que existe entre la equidad y el desarrollo está sujeta a dos aspectos principales: los efectos de la desigualdad de oportunidades cuando los mercados son imperfectos, y las consecuencias de la falta de equidad para la calidad de las instituciones que genera una sociedad. Es decir, los mercados de los países en desarrollo presentan muchas deficiencias, en particular los mercados de crédito, seguros, tierra y capital humano; aunado a esto, el hecho de que un nivel elevado de desigualdad económica y política suele crear instituciones económicas y mecanismos sociales que favorecen sistemáticamente los intereses de quienes tienen más influencia y, en consecuencia, esas instituciones poco equitativas pueden acarrear costos económicos (2). En resumen, la pobre propuesta del BM se reduce a que, para lograr una mayor equidad, hay que crear un clima institucional propicio para la inversión y lograr que los pobres se “empoderen”.

En resumen, el Banco Mundial plantea que es posible el mix público-privado en cuanto atención médica se refiere, acompañado de los seguros privados, siempre y cuando los servicios de salud pública sean garantizados por los poderes públicos. No compartimos esta posición en lo absoluto, debido a que, contrariamente a esta preocupación internacional, las desigualdades, la injusticia social y las inequidades no parecen haber disminuido en forma importante en los países subdesarrollados. Nuestra óptica insiste en el derecho ciudadano a la salud y la responsabilidad del Estado para su protección.

Algunos elementos que definen desigualdad y equidad

Ferrer (3), entre otros autores, sostiene que una de las mayores contribuciones de los estudios sobre desigualdades en salud ha sido identificar la salud como un fenómeno multidimensional, que no depende exclusiva ni mayoritariamente del acceso a los servicios de atención de salud.

Las desigualdades e inequidades sociales en el campo de la salud han sido definidas como "disparidades de salud en un país y entre diferentes países que se consideran improcedentes, injustas, evitables e innecesarias (no inevitables ni irremediables) y que gravan sistemáticamente a poblaciones que han sido hechas vulnerables por las estructuras sociales subyacentes y por las instituciones políticas, económicas y legales”(4). 
De acuerdo con Whitehead (5), las desigualdades o inequidades en salud son injustas cuando no son resultado de la libre elección de los individuos, sino que son producto de situaciones que están fuera de su control. La misma autora señala que las diferencias en las condiciones de salud derivadas de desigualdades sociales son evitables ya que no son diferencias producidas por la biología de los individuos.

Amartya Sen (6) también hace referencia a la justicia y a la equidad en salud y señala que tiene muchos aspectos y lo mejor es verla como un concepto multidimensional. Incluye aspectos relacionados con la capacidad de lograr una buena salud, capacidad que está distribuida desigualmente e implica la capacidad de luchar por la propia salud o la de un colectivo y no sólo con la distribución de la atención sanitaria. Pero también incluye la justicia de los procesos y, por lo tanto se debe prestar atención a la ausencia de discriminación en la prestación de la asistencia sanitaria. Un buen compromiso con la equidad en salud también necesita que las consideraciones sobre la salud se integren en los temas más amplios de la justicia social y de la equidad global, prestando suficiente atención a la versatilidad de los recursos y a las diferencias de alcance e impacto de los diferentes acuerdos sociales. Otro señalamiento establece que es necesario hacer la diferencia entre los logros y la libertad de lograr (freedom to achieve) y que en este terreno, el conflicto real se da entre los diferentes tipos de libertad que disfrutan las personas. En torno al concepto de "desigualdad de oportunidades" señala que debe más bien ser referido a "desigualdad en las capacidades" y sostiene que la sociedad debe ofrecer a sus miembros la posibilidad de aumentar sus capacidades y no sólo otorgarle los bienes que puedan hacerle falta.

Entendemos que esta capacidad forma parte de la categoría de ciudadanía completa en cualquier sociedad, que se desarrolla a partir de la participación ciudadana que conduce a capacitar a la población en la defensa de su derecho a la salud. No es posible que se garantice equidad en la distribución de recursos para la atención a la salud de ningún sistema sin que el control de la política sanitaria esté en manos de la ciudadanía ${ }^{1}$.

Recientemente hemos vuelto a hablar de pobreza como sinónimo de desigualdad, que sin duda lo es, pero quisiéramos recordar la definición de Hannah Arendt (7) "La pobreza es algo más que la carencia; es un estado constante de indigencia y miseria extrema cuya ignominia consiste en su poder deshumanizante; la pobreza es

${ }^{1}$ Cele de A. BR. [Cidadania e direitos: Dilemas da questáo social]. Tesis Doctoral, 1995. Se encuentra en la Fundación Oswaldo Cruz/Escuela Nacional de Salud Pública. Departamento de Ciencias Sociales. Río de Janeiro, Brasil. 
abyecta debido a que coloca a los hombres bajo el imperio absoluto de sus cuerpos, esto es, bajo el dictado absoluto de la necesidad, según la conocen todos los hombres a través de sus experiencias más íntimas y al margen de toda especulación”.

Se trata de reconocer que las determinaciones sociales de la salud se expresan en diferentes perfiles de salud y comprender que la pobreza aunada a una ciudadanía incompleta empeora estas desigualdades en salud.

Para retomar el tema de la equidad podemos mencionar que la falta de equidad en salud es una consecuencia de procesos sociales como ingresos insuficientes, desocupación, un nivel de vida precario, diferencias en función del género, origen étnico y grupo de edad, consecuencias todas de la estructura social. No queremos decir con esto que es necesario cambiar totalmente la estructura social para hacer menos desigual la salud de las poblaciones; pero suponer que la desigualdad y la inequidad en salud desaparecen con los nuevos modelos meritocráticos, que dividen el derecho ciudadano a la salud en aquellos que pueden pagar o tienen un cierto sistema de seguridad social y aquellos que carecen de seguridad social, es soñar con un imposible.

Por otro lado, escasean los estudios sobre formas concretas de la inequidad en servicios de atención; recién en los últimos años han generado interés, dada la gran disparidad en la eficiencia y aún en la calidad de la atención (8). Todo lo anterior se refuerza con las cifras publicadas por la CEPAL (9) que señalan que, en el año 2005, el número de latinoamericanos que vivía en la pobreza alcanzó los 209 millones de personas, de los cuales 81 millones eran pobres extremos, lo que representaba el 39,8 \% y 15,4 \% de la población respectivamente. La situación de desigualdad en todas sus expresiones en América Latina era brutal y sigue siéndolo.

La injusticia social como común denominador

La discusión sobre desigualdad e inequidad se relaciona con algunos valores, entre ellos, el de justicia social; pero lo que es justicia social para algunos, no lo es para otros. Expondremos a continuación algunos autores que reflexionan sobre inequidad y justicia social.

Análisis recientes realizados en el contexto latinoamericano han explorado la desigualdad a partir del concepto de equidad y justicia social de Barr (10) cuyo significado depende de los valores y las perspectivas sociales. Para el análisis de la equidad en las políticas de salud, la diferencia más relevante se encuentra entre el enfoque igualitarista o solidario, que formula los objetivos de 
equidad en términos de igualdad, y el enfoque neoliberal, que los expresa en términos de niveles mínimos y de maximización del bienestar agregado.

El enfoque igualitarista se apoya en el concepto de justicia social de la filosofía comunitaria, que considera la solidaridad y la unidad social como algo bueno en sí mismo (10). Las políticas de salud cuyos objetivos de equidad están expresados en términos igualitaristas se dirigen a la eliminación de las desigualdades en los niveles de salud. En este caso, se tenderá hacia sistemas de salud financiados públicamente, en los que el aporte del ciudadano debe estar relacionado con lo que puede pagar, de acuerdo a su situación socioeconómica, y no con su consumo de servicios de salud. Un ejemplo de este tipo son las políticas que persiguen la universalización de la cobertura del sistema de salud de un país, con independencia de la capacidad de pago.

En relación al tema de la justicia en los servicios, ésta se traduce en el tema de la justa asignación de los recursos en la política sanitaria. Desde la perspectiva de los juristas, Kelsen (11) señala que todas las doctrinas que intentan definir la justicia como principio universal terminan siendo tautológicas, por lo cual opta por la solución de un orden jurídico y una ley acorde con los valores de una sociedad; pero el problema es que pocas veces se puede hablar de valores que comparta el conjunto de cualquier sociedad en tiempos de modernidad. Por otro lado, debe reconocerse que históricamente las leyes han sido en ocasiones absurdas, inhumanas e inmorales. Pensemos como ejemplo en la esclavitud y el nazismo, ambos apoyados impecablemente en la legislación vigente; más aún, en sociedades conflictivas y polarizadas como la mexicana, donde las leyes no siempre resuelven los reclamos sociales y éticos de la población pobre.

Otro teórico neoliberal, Robert Nozick (12), reconoce que la rectificación de las injusticias requiere un Estado "mayor” que el "adelgazado" para resolver los problemas de la justicia distributiva y, en especial, en el caso de la atención a la salud, cuyas injusticias se arrastran de muchos años. De acuerdo con este autor se puede afirmar que el Estado mínimo no puede garantizar la justicia en la atención a la salud.

Nozick (12) y Engelhardt (13) reconocen que los pobres no pueden entrar en el mercado libre y por lo tanto quedan excluidos de la distribución de bienes y derechos para vivir una vida digna y saludable. La solución “racional” es la ayuda filantrópica. 
Baumrin (14) se refiere crudamente a que el derecho a la atención a la salud tiene sólo bases retóricas y casi siempre carece de un sustento económico para cumplirse.

Rawls (15), por su parte, señala que el objeto primario de la justicia es la estructura básica de la sociedad y que el atributo más importante en el reparto de la justicia es la imparcialidad. Ésta se sustenta en principios que serían aceptados por todas las personas libres e iguales que conforman una sociedad; aquí está en cuestión el hecho de que no todas las personas son libres e iguales.

Walzer (16) introduce el concepto de igualdad compleja, que en términos formales significa “que ningún ciudadano ubicado en una esfera o en relación con un bien social determinado puede ser coartado por otra esfera, con respecto a un bien distinto"; es decir, que no se violen los principios de distribución propios de cada esfera y evitar que el éxito en una esfera implique la posibilidad de ejercer la preponderancia en otras. En nuestra opinión, este concepto reproduciría permanentemente la desigualdad y la inequidad.

Si bien reconocemos que el problema de la justicia social no puede ser resuelta en un proceso suma cero (aquel proceso en el cual lo que se le quita a uno se le da a otro), no es menos cierto que las clases dominantes en los países periféricos concentran la mayoría de los recursos en salud, lo cual rompe de partida con la equidad. Pero no es cierto que simplemente pasando cierta cantidad que "le sobra” a los más ricos y aplicándola a los sectores más pobres, se resuelva automáticamente el problema de la equidad.

En resumen, para garantizar la equidad en salud sería necesario establecer diagnósticos de los diferentes perfiles epidemiológicos de nuestros países, generar nuevos modelos de atención a la salud, garantizar el derecho a la salud, garantizar un nivel de conciencia ciudadana sobre el derecho a la salud, rechazar el modelo del "mix público-privado" y garantizar un solo sistema de atención a la salud, con financiamiento público y participación ciudadana. Sin estos elementos, la equidad en salud es imposible y las desigualdades caen en el terreno de la injusticia social.

El uso no diferenciado de desigualdad e inequidad

Desde el inicio señalamos que uno de los objetivos de este trabajo era discutir el mal uso de inequidad y desigualdad como sinónimos. Para empezar revisamos las siguientes definiciones de equidad: 
- Disposición del ánimo que mueve a dar a cada uno lo que merece (17).

- Propiedad por la que la prosperidad económica se distribuye equitativamente entre los miembros de la sociedad (18).

- Cualidad que consiste en dar a cada uno lo que se merece en función de sus méritos o condiciones o cualidad que consiste en no favorecer en el trato a una persona perjudicando a otra (19).

- Cualidad que consiste en atribuir a cada uno aquello a lo que tiene derecho (20).

Si a esto añadimos la discusión que hemos señalado en la sección anterior, podemos afirmar que la equidad tiene que ver con la adecuada distribución de bienes y servicios en la población, y la desigualdad en salud significa la diferencia en los perfiles epidemiológicos de clases y grupos sociales; es decir, es la forma en que se distribuye el proceso salud enfermedad en las poblaciones.

Por otro lado el término de inequidad tiene mayor relación con las políticas sociales y de salud, en el sentido de modelos de atención a la salud, siguiendo las necesidades que plantea la desigual distribución del proceso saludenfermedad.

Proponemos que se reserve el uso de desigualdad en salud al proceso saludenfermedad y a los perfiles epidemiológicos por clase social, y se utilice el término de equidad para aquellos procesos que garanticen una mejor atención a las necesidades de salud en diferentes grupos sociales y que no se refieren exclusivamente a la atención a la salud, sino a las condiciones de vida.

Si estamos de acuerdo con el razonamiento anterior, el máximo de equidad se obtendría en aquella sociedad donde fuera posible responder a las necesidades de los diferentes perfiles epidemiológicos, con servicios adecuados para cada perfil. Esta debería ser la utopía a alcanzar para los que nos preocupa el problema de la equidad/inequidad en salud; pero también insistimos en que la equidad total es insostenible con el capitalismo neoliberal en los países periféricos y, hasta cierto punto, en los centrales también.

En el siguiente apartado intentamos demostrar el grado de desigualdad social al que ha llegado México, y que hace imposible los intentos de equidad, sobre todo en la situación en que la salud se ha transformado en mercancía. 


\section{CASO MÉXICO}

México es el país más obediente y más afectado de toda América Latina por la política neoliberal y el Tratado de Libre Comercio (TLC), lo cual no esconde sus contradicciones internas; todo lo contrario, las ha hecho más evidentes. Como consecuencia, es un país muy polarizado social y económicamente, como se demuestra a continuación.

Figura 1. Ingreso de la población económicamente activa (PEA)

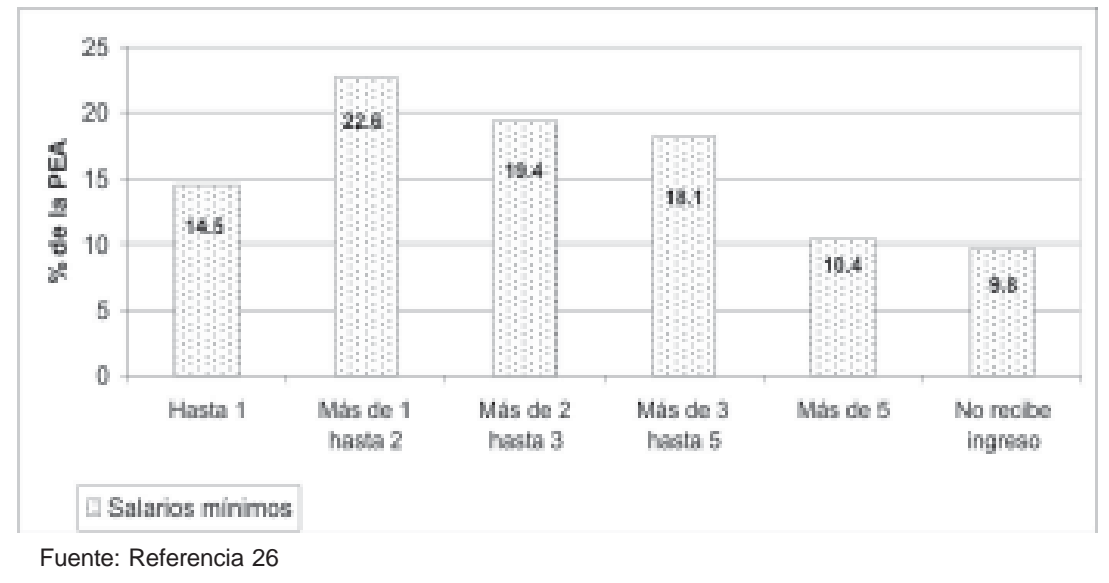

El desempleo en México se ha mantenido alrededor de $4 \%$ a partir de 1994, cuando se firmó el TLC, y a partir de 2003, aumentó hasta tasas de 4,5 \% (la forma de medir este desempleo no considera el subempleo y a los que han desistido de buscar trabajo). En cuanto a la polarización económica, como se ve en la Figura 1, solamente el 10,4 \% más rico de la población recibe ingresos mayores a 5 salarios mínimos (480 USD aprox.), y la dispersión dentro de este grupo 10,4 \% más rico, está entre 5000 y 500000 pesos o más al mes, lo cual significa una concentración del ingreso aterradora. En contraste, 46,9 \% de la población vive con menos de dos salarios mínimos al día, es decir, con 8 USD diarios o menos por 8 horas de trabajo, en un país cuyo costo de vida es el más alto de América Latina. Cabe mencionar que el salario mínimo en Estados Unidos es de 8 dólares la hora para trabajadores no calificados. De allí que no debe extrañar la gran migración ilegal que existe hacia Estados Unidos.

A su vez, en la Figura 2 vemos que el decil X maneja el $40 \%$ de la riqueza nacional mientras que los otros nueve deciles en conjunto disponen sólo del 60 \% de esta riqueza; otra expresión de la concentración desproporcionada de la riqueza. 
Figura 2. Distribución de la riqueza por deciles 2000-2004*

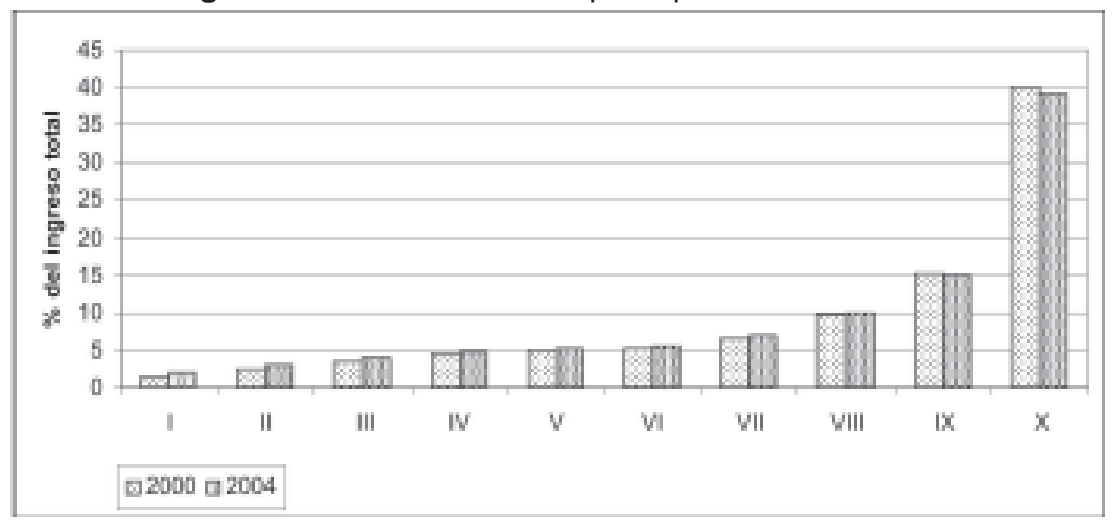

Fuente: Referencia 26. *Por ciento de ingreso por decil, el total de los hogares se ordenan de acuerdo a su ingreso y se dividen en 10 partes proporcionales (deciles). El decil I corresponde a los hogares más pobres y el decil $X$ a los más ricos.

Luis Ortiz en México (datos no publicados) ha hecho un esfuerzo por correlacionar variables que permiten demostrar la gran desigualdad en salud, articulando indicadores oficiales que no fueron creados para demostrar la desigualdad; así, Luis Ortiz pudo construir una correlación positiva entre el bajo nivel educativo de la madre y la mayor desnutrición infantil. En uno de los polos de la desigualdad se encuentran el Distrito Federal y los Estados del norte del país (Nuevo León, Coahuila, Sonora, Sinaloa, Tamaulipas), con los niveles más bajos de analfabetismo de las madres y el más bajo porcentaje de desnutrición en niños, mientras que los valores más altos, tanto de mujeres que no saben leer como de desnutrición infantil, se encuentran en el sureste mexicano, Chiapas, Yucatán, Oaxaca, Guerrero, que son los estados con mayor marginación en el país y con el porcentaje más alto de población indígena.

Por otro lado el mismo autor correlacionó el salario con la mortalidad infantil en mujeres y se encuentra la misma desigualdad entre el Distrito Federal y los estados del norte del país, donde la población ocupada que gana menos de 1 salario mínimo es sólo del 10\% para el DF y del 8.5\% para Nuevo León y la mortalidad infantil en mujeres es entre 13 y 13.5 por mil nacidos vivos para el norte, mientras en Chiapas, Oaxaca y Guerrero, el porcentaje de la población que gana menos de 1 salario mínimo es de 55\% 50\% y 40\% respectivamente, y la mortalidad infantil de mujeres es de 25,24 y 22.8 por mil nacidos vivos, respectivamente. 
Hasta aquí hemos demostrado cómo afecta la polarización de la riqueza nacional a la salud y la educación de la población. A continuación hacemos un análisis de la evolución de la inversión pública en salud.

El sistema de salud en México se caracteriza por el famoso mix públicoprivado, que en realidad ha permitido la privatización creciente del sector salud.

Según información oficial, en México el gasto total en salud representaba para 2005 el 5,7 \% del PIB; cabe aclarar que únicamente menos de la mitad es gasto público; es decir, más del $50 \%$ lo constituye el gasto privado. Si lo comparamos con otros países, encontramos que Canadá tiene un gasto de 9,1 \%, Europa occidental varía entre un 7 y $10 \%$, y en América Latina el promedio de gasto en salud como porcentaje del PIB es de 6,1\%; es decir, México tiene un gasto total insuficiente y, además, centrado en el gasto privado $(21,22)$.

La política de salud en México

En la década de los 80 se iniciaron en México y en otros países de América Latina las reformas sanitarias con la creación de sistemas nacionales de salud, orientadas por las grandes agencias internacionales.

En realidad, la creación de la mayoría de los sistemas nacionales de salud se hizo bajo el paradigma del mix público-privado y, aunque no fue parte explicita del discurso la reforma en México, se caracterizó por la descentralización inequitativa y la privatización de los servicios de atención médica (23).

El eje central de la transformación neoliberal de la política de salud fue en el Instituto Mexicano del Seguro Social (IMSS) y bajo las orientaciones del Banco Mundial.

Con las reformas de 1995 y 1997 se garantizó la no obligatoriedad de aportar al IMSS y se abrió la posibilidad de aportar a empresas de seguros privados. Con esta decisión se rompió con el principio básico de la solidaridad y se privatizó la atención a la salud de los trabajadores de las grandes empresas. En 1997 el IMSS entrega a las empresas privadas (AFORES) el capital de las pensiones manejadas por el Sistema de Ahorro para el Retiro (SAR). Estas y otras medidas de corte neoliberal llevan a la quiebra del IMSS. 
Es importante señalar que en 1998 se firma el acuerdo de préstamo IMSSBanco Mundial, poco conocido hasta la fecha. Se establecieron varias condiciones para el préstamo, entre ellas:

- $\quad$ La separación de las áreas de financiamiento y las de atención médica. Esto es, el "mix público/privado".

- $\quad$ Alentar el financiamiento y la oferta privada de seguros.

- $\quad$ Alentar la prestación de servicios clínicos por el sector privado².

Lo sorprendente es que el cumplimiento de estas condiciones fuera a satisfacción del propio BM, y que el IMSS y el Gobierno Federal obedecieran a pie juntillas éstas condiciones.

Otra condición para el préstamo del BM fue que dentro de la estructura del IMSS se creara una unidad de coordinación del proyecto, financiada por un préstamo extra de 15 millones de dólares y encargada de colaborar en el desarrollo de la reforma. Al mismo tiempo, esta unidad evaluaría la reforma, es decir, sería juez y parte en la nueva política del IMSS.

En resumen, con la reforma sanitaria y con los cambios al IMSS se obtuvo la transformación de la salud en una mercancía; el crecimiento del sector privado, sostenido por los seguros privados de salud, dio por resultado un sistema de atención diferenciado y desarticulado.

Como resultado tenemos en la actualidad que en México se transita de derechos sociales a derechos individuales; de universalidad a propuestas crecientemente selectivas; de solidaridad a individualización de costos y de integralidad a fragmentación. Es decir, se reproduce en forma creciente y permanente la inequidad.

Estado, ciudadanía y equidad en salud

Las políticas de ajuste en México y la obediencia a las medidas neoliberales dejaron un Estado débil y “adelgazado”, por lo tanto, incapaz de regular la irrupción del sector privado, aumentando la inequidad en salud. Todo lo anterior, aunado a la existencia de una ciudadanía incompleta (24), entendida como aquella que no ha tenido oportunidad de ejercer su independencia del gobierno

${ }^{2}$ García RH. La atención a la salud y la justicia distributiva. Tesis de Doctorado en Bioética, presentada en la Facultad de Filosofía y Letras, UNAM; 2006 
y de los partidos, no conoce a cabalidad sus derechos y obligaciones individuales y colectivas frente al Estado, no es capaz de participar en un espacio público (25), ni de controlar las políticas sociales que lo afectan, da como resultado un aumento incontrolable de la inequidad en salud en México.

Una de las vías para una posible solución de la inequidad sería transitar hacia un sistema único de salud con participación ciudadana, donde se recuperaran los valores de la solidaridad y la responsabilidad del Estado en el compromiso por el derecho ciudadano a la salud.

Algunas consideraciones finales

- Retomar la propuesta de que no es lo mismo desigualdad que inequidad.

- Reconocer que en los países periféricos, donde la polarización social ha ido en aumento paralelamente con el empobrecimiento de las mayorías, la política sanitaria de corte neoliberal no hace sino empeorar la equidad en los servicios de salud y aumentar la desigualdad en el proceso salud-enfermedad.

- Reconocer que el capitalismo en términos muy generales es incompatible con la equidad, y más acentuado en el caso de los países periféricos.

- Reconocer que hay que seguir luchando por una mayor equidad de los servicios de salud, pero siempre tener presente la incompatibilidad en última instancia, señalada en el párrafo anterior.

-Contribuir a elevar la calidad de la ciudadanía incompleta para alcanzar una ciudadanía conciente de su derecho a la salud y de la responsabilidad del Estado en garantizarlo

\section{REFERENCIAS}

1. OMS. Monitoring Equity in Health: A Policy-Oriented Approach in Low and middle-Income Countries. Geneva, 1998 [Internet] Disponible en: http://www.consorcio.org/observatorio/alertas.asp. Consultado septiembre de 2007

2. Banco Mundial. Informe sobre el Desarrollo Mundial 2006. Panorama General. Equidad y DesarroIlo. Washington D.C., 2005, [Internet] Disponible en: http://siteresources.worldbank.org/ INTWDR2006/Resources/477383-1127230817535/0821364146.pdf Consultado en octubre 2007

3. Ferrer L. Equidad y justicia en salud. Implicaciones para la bioética. Act Bioet, 2003; (1):113-126

4. Krieger N. Glosario de epidemiología social. Rev Panam de Salud Pública, 2002; 11 (5/6): 480-490 
5. Whitehead M. Whitehead M. The concepts and principles of equity and health. Int J Health Serv 1992;22(3):429-45.

6. Sen A. ¿Por qué la equidad en salud?. Rev Panam Salud Publica 2002;11(5-6):302-9.

7. Arendt H. Sobre la revolución. España, Alianza Editorial; 1988. p.61

8. Sonis A. Equidad y salud. Arch. argent. pediatr, 2001; 99(3): 253-256

9. CEPAL. Panorama social de América Latina 2006. Santiago de Chile, 2006. Disponible en: http:// www.eclac.cl/publicaciones/xml/0/27480/PSE2006_Cap1_Pobreza.pdf, Consultado en septiembre 2007

10. Barr N. The Economics of the Welfare State. 2a. Ed. Oxford: Oxford University Press;1993.

11. Kelsen H ¿Qué es la justicia?, Barcelona, Ariel; 1992, pp. 59- 63, 64-93

12. Nozick R. Anarquía, estado y utopía. México, FCE; 1988, pp. 39-47

13. Engelhardt HT. Bioethics and secular humanism: The search for a Common morality. London SCM Press; 1991, pp. 134-135

14. Baumrin $\mathrm{BH}$. Why there is not right to health care. In: Rhodes $\mathrm{R}$, Batin $\mathrm{M}$, Silvers $\mathrm{A}$. Medicine and Social Justice. Oxford University Press; 2002, pp.78-83.

15. Rawls J. Teoría de la justicia. México, FCE; 2002. pp. 137-167

16. Walzer M. Las esferas de la justicia. Una defensa del pluralismo y la igualdad. México: FCE; 2001. p.33

17. Diccionario de la Real Academia de la lengua española, $22^{\mathrm{a}}$ ed. [Internet] Disponible en http:// www.rae.es/ Consultado en octubre 2007

18. Enciclopedia Wikipedia, [Internet] Disponible en http://es.wikipedia.org/wiki/Equidad Consultado en octubre de 2007

19. Diccionario ilustrado de la lengua, Vox, Barcelona; 2002 (s/p)

20. Diccionario Pequeño Larousse llustrado, Larousse, México; 2003, p.398

21. Secretaría de Salud. Salud: México 2001-2005. Información para la rendición de cuentas. México, 2006. [Internet] Disponible en http://evaluacion.salud.gob.mx/saludmex2005/ sm2005.htm Consultado en septiembre de 2007

22. Ortiz HL, López MM, Rosales FRA, Rivera MJA, Laurell AC. Mortalidad infantil y desigualdad socioterritorial en México. México, UAM. Serie Académicos CBS, 38; 2002. pp.12-15

23. Eibenschutz C, Vilar E. Políticas sanitarias. Historia, situación actual y propuesta de cambio. En Calva JL (coord.) Derechos y políticas sociales. Agenda para el desarrollo, Vol.12, México, UNAM-Miguel Ángel Porrúa; 2007. pp.192-203.

24. Eibenschutz C. México: Gobierno autoritario, ciudadanía incompleta. LASA 2000, XXII Congreso Internacional. [Internet] Disponible en http://lasa.international.pitt.edu/Lasa2000/ Eibenschutz.PDF. Consultado en Agosto de 2008

25. Habermas J. The Structural Transformation of the Public Sphere. England: Polity Press; 1996, pp.31-43.

26. Aguayo QS. Almanaque Mexicano 2007. México: Aguilar; 2007. p.79. 